\title{
Neural basis for successful encoding and retrieval of prospective memory
}

\author{
CHEN YanNi ${ }^{1}$, GUO ChunYan ${ }^{1,3 *} \&$ JIANG Yang ${ }^{2}$ \\ ${ }^{1}$ Beijing Key Laboratory of Learning and Cognition, Department of Psychology, Capital Normal University, Beijing 100048, China; \\ ${ }^{2}$ Department of Behavioral Sciences, College of Medicine, University of Kentucky, Lexington KY 40536-0086, USA; \\ ${ }^{3}$ Key Laboratory of Mental Health, Chinese Academy of Sciences, Beijing 100101, China
}

Received December 5, 2010; accepted April 4, 2011

\begin{abstract}
Prospective memory (PM) refers to memory for future intentions. Difference due to memory (Dm effect) is the difference in neural activity related to stimuli that were subsequently remembered or forgotten. Using event-related potentials (ERPs), the present study investigated the Dm effect for PM using a subsequent task-switching paradigm. The results showed that a Dm effect of ERP P150 was more positive-going for later PM hit trials than for later PM forgotten trials during 100-200 ms. This $\mathrm{Dm}$ effect may reflect the process for the production of future intention or the process for attention. Consistent with previously reported Dm effects of other types of memory, we found that the fbN2 (250-280 ms) and late positivity component (400-700 $\mathrm{ms}$ ) were stronger in later PM hit trials than in forgotten trials. The fbN2 was evoked by Chinese characters. The late positivity component was related to the precise encoding process. In conclusion, because of the early P150, PM encoding appears to be somewhat different from previously identified Dm effects. However, further research is needed. Our findings reveal that Dm effects of PM share similar characteristics with known Dm effects of other types of episodic memory after the very early stage of neural processing.
\end{abstract}

difference due to memory effect, prospective memory, event-related potential, P150, late positivity component

Citation: $\quad$ Chen Y N, Guo C Y, Jiang Y. Neural basis for successful encoding and retrieval of prospective memory. Sci China Life Sci, 2011, 54: 580-587, doi: $10.1007 / \mathrm{s} 11427-011-4176-8$

Prospective memory (PM) is a type of episodic memory, defined as remembering to perform intended actions. In contrast to retrospective memory $(\mathrm{RM})$ that recalls past events and external stimuli, PM refers to remembering to carry out intended actions in the future that are represented internally. PM has three main characteristics: a delay between encoding and the intended action, an ongoing task in the delay, and self-initiated intention execution [1]. A task-switching approach [2] for studying PM involves asking participants to remember to press a key on a keyboard (e.g., "D") whenever they see a particular item (e.g., red words) in the context of an ongoing task (e.g., tone judg-

*Corresponding author (email: guocy@ hotmail.com) ment). PM tasks include four cognitive phases: intention formation, intention retention, intention initiation, and intention execution [3]. Forming an intention is an essential phase for successful PM and is also a prerequisite of PM performance [4]. Three components must be encoded in the intention formation phase: the retrieval criterion, the to-beperformed action, and the decision [5]. Zöllig et al. [4] proposed that forming an intention requires not only RM processes, but also executive functions. As an example of these components, suppose that I sit in a classroom with the intention to buy a textbook when I go past the book store on my way home. RM processes involve the time at which the PM response has to be performed (i.e., past the book store). Executive functions involve planning and coordinating the 
decision to act (i.e., buying the textbook).

Since the concept of PM was put forward, the majority of research has focused on specific processes or group differences during the retrieval phase [6-14]. However, there are limited studies examining the neural basis of the PM encoding phase using brain imaging techniques, e.g., eventrelated potentials (ERPs), functional magnetic resonance imaging (fMRI), and positron emission computed tomography (PET). A previous fMRI study on the encoding of future intentions (PM) and actions (RM) showed that PM activated the temporal lobe including the hippocampus (predicting overall memory success), and some additional regions, including left rostrolateral $\mathrm{PFC}$ and the right parahippocampal gyrus (recruited by future intention processing) [15]. The results provided evidence that PM encoding has both a common episodic memory network and an executive network specifically recruited by an intention. ERP was used to investigate the PM encoding mechanism [4,10,11]. West and colleagues [10,11] found that a late positivity component (LPC) and frontal-polar slow wave (FPSW) modulated intention formation in adult PM. Researchers have shown that LPC differentiated the ERPs elicited by "memory hit" intention trials and "forgotten" trials from those elicited by baseline activities or differential LPCs between "hit" intention trials and the baseline [4]. The "hit" intention trial was associated with a more positive LPC compared with that of the "forgotten" trial in the parietal region. Curiously, the LPC polarity reversed in lateral frontaltemporal regions. The parietal LPC was probably a P3-like response, and the frontal LPC reversal was attributed to the method of ERP [11]. Moreover, Zöllig et al. [4] found that the LPC was different among adolescents, young adults, and old adults, indicating that they adopted different intention encoding strategies. The FPSW differentiated the ERPs elicited by "hit" intention trials from "forgotten" trials [10,11], or differentiated the ERPs elicited by "hit" intention trials from those elicited by the baseline [4]. The FPSW showed a sustained negativity over the fronto-polar region, which reflected the neural correlates of elaborative encoding processes only in adolescents and adults [11]. Although the polarity was reversed, they believed it resembled subsequent memory effects (Sm; Dm) [16,17]. The Dm effect refers to the neural activity difference between stimuli that were subsequently remembered or forgotten. However, in many Dm effect studies, the P300 or LPCs for later-remembered items were more positive than those for later-forgotten ones [18].

Although these results are extremely valuable, knowledge gaps remain in PM encoding studies. One way to study memory encoding is to monitor brain electrical activity with the ERP technique. The neurophysiological correlates of the Dm effect provide a way to indicate retrieval performance during the encoding phase. Although previous studies found some ERPs (LPC, FPSW) associated with PM encoding, they did not compare the Dm effect in
PM to that in episodic memory. PM is a special kind of episodic memory, with the relationship between them still unclear. The numbers of "forgotten" ERP trials were often low in these experiments $[10,11]$. ERPs require multiple trials to extract brain signals from background brain activity (approximately 15 trials are required to form a reliable ERP average for an individual participant) [19]. Additionally, they did not distinguish the components of intention formation [5]. Poppenk et al. [15] performed an fMRI experiment to investigate PM encoding from the identification of PM cues. The scope of our study was also limited to the identification of PM cues (Chinese two-character words).

The present experiment aimed to investigate the Dm effect of PM and its relationship with known Dm effects using the ERP technique. The procedure was similar to that used by West and Krompinger [13], with modifications such as a distraction task following the encoding phase to prevent subjects from repeating, low-frequency Chinese two-character words as stimuli, and different presentation duration and ITI of stimuli. The most distinctive difference was that the aim of this study was to explore both the encoding and retrieval phases. We mainly focused on the PM encoding phase and compared the ERPs for later PM hit trials with those of later PM forgotten trials. According to an fMRI study, PM encoding is supported by both a common episodic memory network and an executive network specifically recruited by future-oriented intention [15]. Therefore, the hypothesis of this ERP study was that there might be a unique ERP component for PM encoding (associated with intention and frontal planning) which differentiates a Dm effect from the Dm effect related to episodic memory. This study provided evidence from ERPs regarding the mechanism involved in the encoding phase of PM.

\section{Methods}

\subsection{Participants}

Fifteen right-handed students ( 7 males and 8 females; mean age $=22$ years, range $=18-24$ years) participated in the experiment. All participants could speak standard Mandarin. They were healthy, with normal or corrected-to-normal vision. They were provided with written and oral descriptions of the study before written informed consent was obtained.

\subsection{Stimuli}

The experimental materials included 1440 Chinese twocharacter words (range $=2-5$ occurrences/million) [20]. Of them, 192 pairs were selected as similar lexical words (one character was the same, the other was different). The rest were used in an ongoing task. The similar lexical words were divided into two groups, with 96 pairs used in the 
ongoing activity phase and divided into two sets as PM cues and PM lures. The remaining 96 pairs were used in a recognition task and were divided into two sets as RM cues and new items. Half of all the words were composed of two characters sharing the same tones; the other half were different. The words were matched on tone of the Chinese characters, familiarity, and visual complexity. Any polyphones were eliminated.

\subsection{Procedure}

Subjects sat comfortably in front of a computer monitor in a sound-proof room with dim light. All stimuli were shown at the center of the screen against a black background. The font used was 60-point SimSun. Subjects were requested to fixate their eyes on the center of the screen. A fixation cross subtending a visual angle of $1.86^{\circ} \times 4.37^{\circ}$ was presented in the center of the screen before each trial began, and the ITI lasted $(900 \pm 200) \mathrm{ms}$. Each trial was presented pseudo randomly. Each of the 48 blocks of the memory task included three phases, as shown in Figure 1. Each phase (Encoding, Same/Different trials, Recognition) was preceded by instructions indicating the actions in the next phase of the block to be performed. The encoding phase included two PM cues (in red) for a later PM test and two RM cues (in blue) for a later recognition test that were presented sequentially. All the cues were presented for $500 \mathrm{~ms}$. In this phase, subjects were asked to memorize all the presented words. The distraction task followed the encoding phase, in which participants were asked to subtract 3 from a 3-digit number shown on the screen for $3000 \mathrm{~ms}$. In the ongoing activity phase, each trial was presented in white against the black background for $1300 \mathrm{~ms}$. There were three kinds of words including two PM cues which were studied in the encoding phase, two PM lures, and 22 tone judgment trials (11 the same and 11 different).

Participants were required to judge whether the tones of two characters were the same (press the "F" key) or not

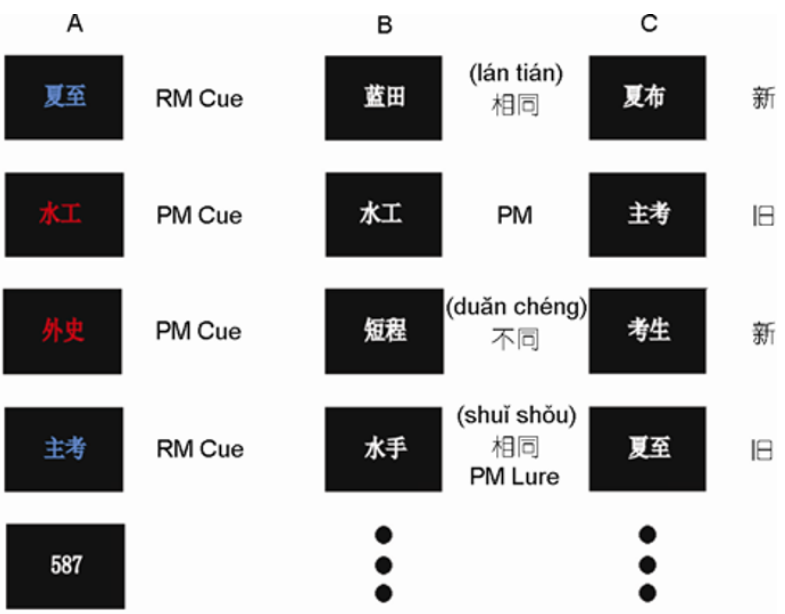

Figure 1 Experimental paradigm. (press the "J" key) for the ongoing trials and the PM lure trials. PM lures were to ensure that subjects responded based on recognizing PM cues, not on perception. For PM cues, participants were required to do a PM response (press the "D" key). PM cue and lure trials were presented on trials 6-25 and as separated by a minimun of five tone judgment trials. The apperance of PM cues before and after the PM lures was conterbalanced. The recognition phase following the ongoing activity phase included four trials, two of which were the RM cues from the encoding phase. The others were similar words (new). Subjects were required to discriminate old items (press the "J" key) from new items. Before the task began, individuals were shown a flow diagram that outlined the structure of the task and what to do during each phase of the task.

In the encoding phase, subjects attempted to memorize red words (prospective memory cues) for a later prospective memory test and blue words (retrospective memory cues) for a later recognition test. In the ongoing activity phase, there were three kinds of words including PM trials, PM lures and ongoing trials. Subjects judged whether the tones of the words were the same for ongoing trials, and PM lures. For PM trials, however, the correct response was a PM response. In the recognition phase, subjects were asked to discriminate old (RM trials) from new items.

\subsection{Acquisition and analysis of electrophysiological data}

Electroencephalographic recordings were made from 62 scalp sites using $\mathrm{Ag} / \mathrm{AgCl}$ electrodes embedded in an elastic cap of ESI-64-channel EEG recording system. Vertical electrooculographic (EOG) signals were recorded by electrodes placed directly above and below the supra-orbital area of the left eye. Horizontal EOG and blinks were monitored via a bipolar montage at the outer canthi of both eyes. A left mastoid reference electrode was used online, and the reference was changed offline to the average of left and right mastoid recordings. The ground electrode was between $\mathrm{FPz}$ and Fz. EEG signals were filtered with a band-pass of $0.05-40 \mathrm{~Hz}$ and sampled at a rate of $500 \mathrm{~Hz}$. Impedance was less than $5 \mathrm{k} \Omega$. EEG recordings were averaged off-line. Ocular artifacts were reduced, and trials with a voltage exceeding $\pm 75 \mu \mathrm{V}$ at any electrode were excluded from analysis as artifacts.

ERPs were quantified by measuring mean amplitudes in several latency intervals: (100-200, 250-280, and 400-700 ms for the encoding (-100-900 ms) phase, and 200-400 and $400-900 \mathrm{~ms}$ for the retrieval ( $-100-1200 \mathrm{~ms})$ phase relative to the mean amplitude of the pre-stimulus baseline $(-100-0 \mathrm{~ms})$. These intervals were selected based on the visual inspection of grand-average ERPs, given that similar intervals have been used in prior studies of related ERP phenomena [4,13,21]. ERPs were averaged for artifact-free trials associated with later PM hit $(M=51.00)$ and later PM forgotten trials $(M=37.73)$ in the encoding phase; and PM hit $(M=50.93)$, 
PM miss $(M=39)$, and ongoing activity trials $(M=787.13)$ in the retrieval phase. Because the number of RM miss trials was very small (range was 12-45 trials, average was 23 , and generally in our laboratory the number of trials must be more than 25), and this study aimed to explore the PM Dm effect, we only report the analysis of data for PM, not for RM. ERPs were averaged across all 15 subjects from 21 locations including anterior/posterior (frontal pole, frontal, fronto-central, central, centroparietal, parietal, occipital) and lateral (left, middle, right) locations. The 21 scalp sites included on the left FP1, F3, FC3, C3, CP3, P3, and O1; at midline $\mathrm{FPz}, \mathrm{FCz}, \mathrm{Fz}, \mathrm{Cz}, \mathrm{CPz}, \mathrm{Pz}$, and $\mathrm{Oz}$; and on the right FP2, F4, FC4, C4, CP4, P4, and O2. ERP measurements were evaluated using SPSS 13.0 software (manufacturer is SPSS Inc., 233 South Wacker Drive, Chicago). The Greenhouse-Geisser correction was applied to adjust the $P$-values $(P<0.05)$ that were calculated by repeatedmeasures analysis of variance (RM-ANOVA) tests with different factors.

\section{Results}

\subsection{Behavioral results}

Results for the mean accuracy and response time (RT) for PM trials, PM lure trials, and ongoing trials in the ongoing activity phase are shown in Table 1. PM cues elicited a correct prospective response ("D" key); PM lures and ongoing trials were generally associated with a tone judgment (same "F", different "J"). The results showed that the main effect of response accuracy was significant, $F(2,28)=62.85$, $P<0.01$. Post hoc multiple comparisons suggested that accuracy for PM trials was lower than those for PM lure trials and ongoing trials $(P s<0.01)$. Accuracy for PM lure trials was lower than for ongoing trials, $P<0.05$. RT was also significant, $F(2,28)=54.60, P<0.01$. Post hoc analysis showed that RT for PM hits was shorter than for PM lure hits and ongoing hits $(P s<0.01)$. However, RT for PM lure hits was longer than for ongoing hits, which showed that PM lures consumed more attentional resources or initiated rehearsal of the intention.

\subsection{ERP results from the study phase}

To examine the Dm effect, we compared ERPs of later PM hit trials with later PM forgotten trials in the encoding phase (Figure 2). Three modulations of ERPs were selected: P150

Table 1 Mean accuracy (percentage) and response time (ms) in the ongoing activity phase

\begin{tabular}{ccc}
\hline & Accuracy $(\mathrm{SE})$ & Reaction time $(\mathrm{SE})$ \\
\hline PM trials & $57 \%(2.67)$ & $1046(39.51)$ \\
PM lure trials & $74 \%(1.96)$ & $1328(49.65)$ \\
Ongoing trials & $83 \%(2.17)$ & $1250(44.51)$ \\
\hline
\end{tabular}

A
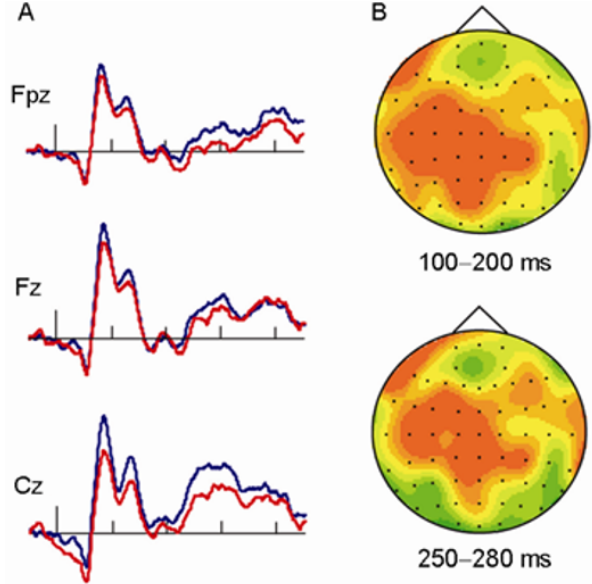

100-200 ms
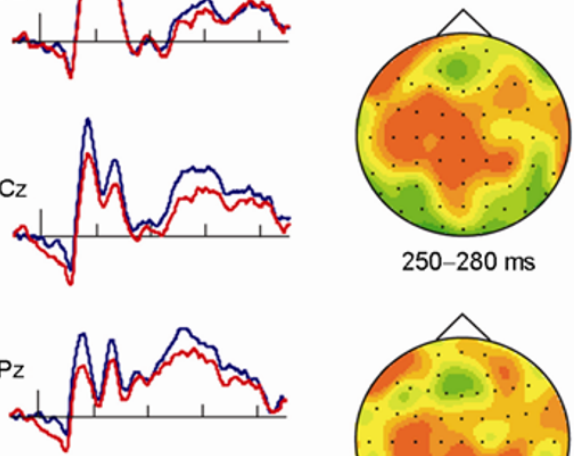

$\mathrm{Oz}$
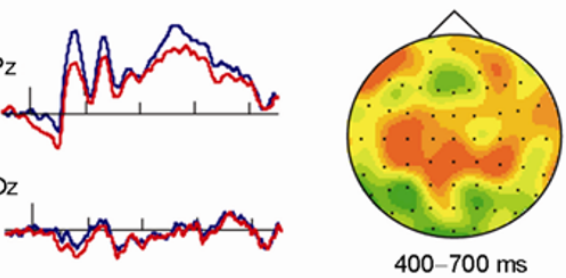

$+2 \mu \mathrm{V}$

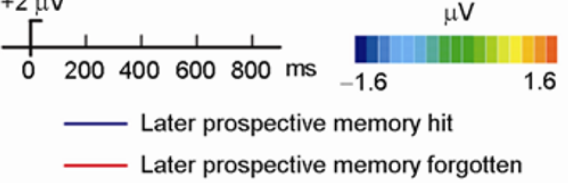

Figure 2 The Dm effect of prospective memory.

(100-200 ms), fbN2 (250-280 ms), and LPC (400- $700 \mathrm{~ms})$. A $2 \times 7 \times 3$ RM-ANOVA with three factors, Dm (later PM hit, later PM forgotten), anterior/posterior location, and laterality, was conducted on mean amplitude data.

During the latency of P150, the main effect of Dm reached statistical significance, $F(1,14)=11.96, P<0.01$, with the amplitude of P150 being greater for later PM hit items than for later PM miss items. Interestingly, the interactions of Dm by anterior/posterior location, $F(6,84)=4.08$, $P<0.05$, and location by laterality interactions, $F(12168)=$ $3.53, P<0.05$, were also significant, meaning that the form of Dm effects was different in different brain regions. Furthermore, a $2 \times 3$ RM-ANOVA with two factors (Dm and laterality) was carried out. Results showed that the main effects of Dm were all significant from frontal pole to occipital electrodes $(P s<0.05)$, indicating more positive responses for later PM hits than those of later PM forgotten trials. The DM effect mainly existed in central, centroparietal, and parietal regions. However, there was no interaction of Dm by laterality. The Dm main effects in the remaining two intervals (250-280 ms, 400-700 ms) were significant (fbN2, $F(1,14)=4.60, P<0.05$; LPC, $F(1,14)=7.39, P<0.05)$, indicating that the ERPs were more positive and stronger for later PM hit trials than those of later PM forgotten trials. The interactions of $\mathrm{Dm}$ by the anterior and posterior region for fbN2 and LPC were not significant $(P s>0.05)$. 
Unexpectedly, the ERPs for PM displayed a very early subsequent memory effect. As illustrated in Figure 2, PM cues that were subsequently remembered elicited a more positive-going ERP almost immediately after their onset $(\mathrm{Pz})$. The $2 \times 7 \times 3 \mathrm{RM}-\mathrm{ANOVA}$ at $0-100 \mathrm{~ms}$ showed that the interaction between Dm, anterior/posterior location, and laterality was nonsignificant, $F(12,168)=1.60, P>0.05$. However, the main effect of Dm was marginally significant, $F(1$, $14)=4.61, P=0.05$. This phenomenon has been reported in subsequent memory for nonwords [22].

A, During the encoding phase, ERP waveforms for later prospective memory hit trials (blue) and later prospective memory forgotten trials (red) are shown on the left; B, topographic maps for differential amplitudes between later prospective hit and later prospective forgotten are presented in three time intervals (right column).

\subsection{ERP results from the retrieval phase}

The Dm effect was based on later memory performance, thus we briefly reported the ERPs for the retrieval phase to explore the differences among PM hit, PM miss, and ongoing trials (Figure 3). This phase was evaluated by measuring mean amplitudes at 200-400 ms and 400-900 ms, capturing the N300 and positive component, respectively. A series of RM-ANOVAs that included three factors, item type (PM hit, PM miss, and ongoing activity trials), anterior/posterior location, and laterality were conducted.

The RM-ANOVA at 200-400 ms (N300) demonstrated a main effect of item type, $F(2,28)=12.27, P<0.01$, and significant interactions between item type and location $(F(12,168)=3.93, \quad P<0.05)$, and location and laterality, $F(12,168)=3.04, P<0.05$. Furthermore, a $3 \times 3$ RM-ANOVA with two factors (item type and laterality) was carried out. The analysis showed that the main effect of item type was significant in all locations $(P s<0.05)$, indicating that ERPs were less negative for PM hit trials and PM miss trials than ongoing trials in central, centroparietal, parietal, and occipital regions. At 400-900 ms, the ANOVA revealed a main effect of item type, $F(2,28)=19.31, P<0.01$. Moreover, interactions between item type and location, and location by laterality were also revealed, $F(12,168)=86.73, P<0.01$; $F(12,168)=7.16, P<0.01$. Furthermore, the $3 \times 3$ RMANOVA showed that the main effect of item type was significant in all locations $\left(P_{S}<0.05\right)$, reflecting a greater positive component for PM hit trials than ongoing trials in the frontal region (frontal positivity), and a greater positive component for PM hit trials than PM miss trials or ongoing trials in other regions (prospective positivity). This positive component in frontal and partial was called frontal positivity and prospective positivity by West. The difference in ERPs in the retrieval phase was consistent with a previous study in which recognition success was associated with brain potentials distinct from those associated with failure [23].

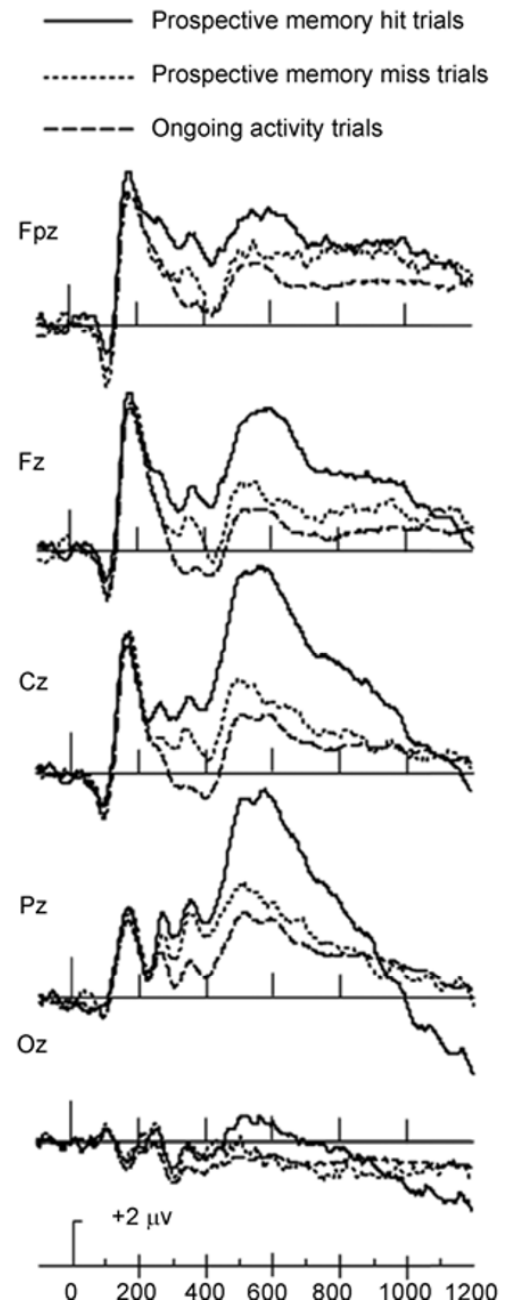

Figure 3 Grand-averaged event-related potentials at select electrodes, demonstrating N300 (200-400 ms), frontal positivity, and prospective positivity (400-900 ms) for prospective memory hit trials, prospective memory miss trials, and ongoing activity trials during the retrieval phase.

\section{Discussion}

\subsection{Dm effect of PM}

The current study aimed to investigate the neural correlates of successful PM encoding. According to subsequent PM performance, we analyzed the Dm effect of PM. The accuracy for PM cues was lower than those for PM lures and ongoing trials, while the response for PM cues was faster than those for PM lures and ongoing trials. These results are consistent with previous research $[9,13]$. The low accuracy rate for PM cues could be attributed to the necessity for self-initiated processes [16,24]. RT for PM hits was faster than RT for ongoing and PM lure trials, which verifies the intention-superiority effect on PM [25] or can be attributed to the recognition of PM cues being simpler than the task of tone judgment. Accuracy for PM lures was lower than that for ongoing items, differing from results reported in previous studies, possibly because the words were semantically 
related to each other in those studies $[13,26]$. However, in the present study, there was structural similarity between characters in word pairs. Moreover, the false alarm rate indicating that PM lures were judged as PM cues was 0.07. RT for PM lures was slower than that for ongoing trials, consistent with evidence from a number of other studies $[9,13,26]$. Some researchers have indicated that the longer response time for lures could be attributed to more attention resources involved in the processing of lures [9,26]. Others explain that lures may initiate rehearsal of the intention [27].

Other than the early ERP component (P150), ERPs for later PM hit trials were more positive and larger than those for later PM forgotten trials, consistent with previous Dm studies [28,29]. Paller and Wagner [29] held the view that encoding depends on at least two components. One component mediates the transformation of sensory input into internal representations. The other component binds the internal representations into an encoding trace [29]. Moreover, the "spontaneous activation theory" proposed by McDaniel and Robinson-Riegler [30] states that PM is mediated by a spontaneous associative memory system and a memory trace encoding mechanism of PM. Therefore, later PM hit trials produce stronger internal representations or deeper memory traces which lead to corresponding changes in EEGs.

The early Dm effect indicating that P150 was more positive for later PM hit trials than those for later PM forgotten trials may be associated with the encoding of PM. First, according to preparatory attentional and memory processes theory [16], successful event-based PM is capacity-consuming. Later PM hit trials may consume more resources than later PM forgotten trials, which evoke changes in early ERP components. Second, evidence from other studies indicates that successful intention is associated with two significant patterns of neural activities: A general episodic memory network involving the temporal lobe, and an executive network linked specifically to PM including left rostrolateral PFC and the right parahippocampal gyrus [15]. On the basis of our topographic results (Figure 2B), we conclude that PM encoding activates the left frontal and central-posterior regions, broadly distributed within 100-200 ms. Leynes et al. [19] found that ERPs elicited by actions for which participants should have established some intentionality to perform the task were more positive than ERP activity elicited by memorized tasks, particularly at right frontal electrode sites. They believed that this does not merely reflect differences in the ability to remember, but rather reflects the cognitive processing associated with establishing an intention to perform an action later. Okuda et al. [31] argued that areas within the frontal lobes (i.e., the left frontal pole and right ventrolateral prefrontal cortex) mediate memory for intentions. That is, subjects produce an intention for a future PM response when meeting PM cues, but later PM forgotten trials do not elicit this intention. Therefore, the more positive responses for later PM hit trials relative to later PM forgotten trials may be due to an intention for a future PM response. This is consistent with Poppenk et al. [15] who concluded that PM has a frontal network linked specifically to PM. This ERP evidence suggests that PM encoding is somewhat different from other types of episodic memory. Third, many Dm studies have found that positive potentials over parietal regions are greater for later-remembered items than those for later-forgotten items during approximately 400-800 ms [28,32], but do not appear early. However, the neural mechanism of attention is often reflected in early ERP components (N1 or P1). The appearance of this Dm effect was very early. Therefore, further studies are needed to verify if this component is associated with PM encoding.

The fbN2 and LPC components have been previously reported related to episodic memory encoding [21,31,32]. The fbN2 component has also been reported in previous studies using Chinese characters as stimuli. Guo et al. [21] used ERPs to monitor the encoding of source memory and item memory processes with Chinese words. They found that there was a Dm effect in the early 220-280 ms interval in the frontal region. They speculated that this early Dm reflected some aspect related to the processing of Chinese words. The present results add evidence to their speculation. However, our distribution of fbN2 elicited by PM was broader than that in their study. Thus, it is possible that different types of memory may be allocated to different psychological resources, causing the differential scalp distribution of Dm effects.

The current ERP results reveal that LPC reflected positive ERPs over central and parietal regions from 400 to $700 \mathrm{~ms}$, consistent with previous studies on episodic memory [32-34]. Many Dm researchers have found that ERPs for later-remembered items are more positive than those for later-forgotten items in approximately $400-800 \mathrm{~ms}$, which sometimes has been attributed to ERP components such as P300 or LPC. A favored cognitive hypothesis regarding these Dm findings is that they reflect superior elaborative encoding for later-remembered items [32]. Paller et al. [35] found that the Dm effect was significant in $\mathrm{Fz}, \mathrm{Cz}$, and $\mathrm{Pz}$ electrodes from 400-700 ms. Yovel and Paller [36] conducted a Dm effect study using face stimuli. They found that recollection Dm was significant at 400-800 ms, whereas familiarity Dm was marginal at 600-800 ms. Guo et al. [33] found that the Dm effects for source memory and item memory were in the latency from 400-600 ms. The LPC in this study resembles the FPSW in previous studies that reflects greater negativity for the ERPs, which were evoked by "hit" intention trials more than by "forgotten" intention trials. In West et al. $[9,11]$, although the FPSW polarity was opposite to that found in other previous studies, they believed that similar cognitive operations are supported by distinct neural systems in the areas of PM and RM. In the current study, however, LPC polarity was consistent with previous studies, except for West et al. [9,11]. Our results 
support the view of Poppenk et al. [15] that PM encoding is supported by a common episodic memory network. Our findings are also consistent with Zöllig et al. [4], namely, that intention formation contains a retrospective component. Therefore, the present findings, particularly in late ERP responses, indicate that the memory mechanisms underlying PM encoding are largely similar to those of other kinds of memory.

\subsection{Retrieval phase}

ERP modulations associated with PM in this study are similar to those observed in previous research. PM hits elicited $\mathrm{N} 300$, frontal positivity, and prospective positivity $[9,10,13]$. The N300 reflected a phasic negativity over the occipital-parietal region 300-500 ms after stimulus onset. This modulation of the ERPs has been associated with the detection of PM cues $[10,11]$. The prospective positivity reflected a sustained positivity over the parietal region between 500 and $1000 \mathrm{~ms}$, and positivity in the frontal area [9]. These positive components (Figure 3 ) have been associated with the neural mechanism that supports the retrieval of an intention from memory [10]. In West's studies, the N300 tends to be largest in amplitude at the lateral PO electrodes rather than at midline, and the P3b seems to be weaker. West suggested that our differences could be based on the reference we used. (i.e., mastoid instead of average). The prospective positivity was also similar to the LPC associated with the recovery of information from episodic memory (i.e., old-new recognition effect) [37-40].

\subsection{Limitations and future directions}

Intriguingly, PM elicited a subsequent memory effect shortly after item onset. It was unexpected and approached marginal statistical significance. This effect has been reported in nonword subsequent memory [22]. It may reflect the benefit to memory of an increased level of attention to PM cues, which would be expected to modulate early ERP components. Given that the time began at $0 \mathrm{~ms}$ and the four words were presented sequentially in the encoding phase, another possibility is that the ITI between them was not long enough. The effect may have been influenced by the pre-stimuli. Therefore, further study will be conducted using task cues prior to each memory cue to determine how subjects respond to a longer ITI. Using task cues will perhaps also result in a recognition test with similar foils so that performance is not too high. Thus, we can compute the RM Dm and then directly compare the PM Dm with the RM Dm.

In summary, the present results add to the literature on Dm effects in several ways. We found that the Dm effect of PM is slightly different from previously recognized Dm effects given that the P150 elicited by later PM hit trials was more positive than that evoked by later PM forgotten trials.
To the best of our knowledge, this Dm effect, which has not been reported previously, may reflect the process of future intention or attentional processes. However, we did not directly compare it with a known Dm in this experiment. Further studies are needed to test such an idea. We also found that the fbN2 ((250-280) ms) and LPC ((400-700) ms) were consistent with known DM effects of episodic memory encoding. In addition, this research consistently revealed two components or modulations of the ERPs that are associated with prospective remembering (i.e., N300, frontal and prospective positivity). The N300 is associated with the detection of PM cues, and the positivity component reflects the activity of a neural mechanism that supports the retrieval of an intention from memory.

This work was supported by the National Natural Science Foundation of China (Grant No. 30870760), Ministry of Education of China (Grant No. 20101108110004), and the Key Foundation of Beijing Municipal Commission of Education (Grant No. KZ201010028029) to Guo ChunYan. The authors gratefully acknowledge R. West (Iowa State University, USA) for helpful discussion at the design stage of the study.

1 Einstein G O, McDaniel M A, Thomas R, et al. Multiple processes in prospective memory retrieval: Factors determining monitoring versus spontaneous retrieval. J Exp Psychol Gen, 2005, 134: 327-342

2 Bisiacchi P S, Schiff S, Ciccola A, et al. The role of dual-task and task-switch in prospective memory: Behavioural data and neural correlates. Neuropsychologia, 2009, 47: 1362-1373

3 Kliegel M, Martin M, McDaniel M A, et al. Complex prospective memory and executive control of working memory: A process model. Psychologische Beiträge, 2002, 44: 303-318

4 Zöllig J, Martin M, Kliegel M. Forming intentions successfully: Differential compensational mechanisms of adolescents and old adults. Cortex, 2010, 46: 575-589

5 Ellis J A, Freeman J E. Ten years on: Realizing delayed intentions. In: Kliegel M, McDaniel A, Einstein G O, Eds. Prospective memory: Cognitive, neuroscience, developmental and applied perspectives. New York: Lawrence Erlbaum Associates, 2008. 1-27

6 Burgess P W, Quayle A, Frith C D. Brain regions involved in prospective memory as determined by positron emission technology. Neuropsychologia, 2001, 39: 545-555

7 Simons J S, Schölvinck M L, Gilbert S J, et al. Differential components of prospective memory? Evidence from fMRI. Neuropsychologia, 2006, 44: 1388-1397

8 West R, Herndon R W, Ross-Munroe K. Event-related neural activity associated with prospective remembering. Appl Cogn Psychol, 2000, 14: $115-126$

9 West R, Herndon R W, Crewdson S J. Neural activity associated with the realization of a delayed intention. Cogn Brain Res, 2001, 12: $1-10$

10 West R, Ross-Munroe K. Neural correlates of the formation and realization of delayed intentions. Cogn Affect Behav Neurosci, 2002, 2: $162-173$

11 West R, Herndon R W, Covell E. Neural correlates of agerelated declines in the formation and realization of delayed intentions. Psychol Aging, 2003, 18: 461-473

12 West R, Wymbs N, Jakubek K, et al. Effects of intention load and background context on prospective remembering: an event-related brain potential study. Psychophysiology, 2003, 40: 260-276

13 West R, Krompinger J. Neural correlates of prospective and retrospective memory. Neuropsychologia, 2005, 43: 418-433

14 West R, McNerney M W, Travers S. Gone but not forgotten: The effects of cancelled intentions on the neural correlates of prospective 
memory. Int J Psychophysiol, 2007, 64: 215-225

15 Poppenk J, Moscovitch M, McIntosh A R, et al. Encoding the future: Successful processing of intentions engages predictive brain networks. NeuroImage, 2010, 49: 905-913

16 Smith R E. The cost of remembering to remember in event-based prospective memory: Investigating the capacity demands of delayed intention performance. J Exp Psychol Learn Mem Cogn, 2003, 29: 347-361

17 Paller K A, Kutas M, Mayes A R. Neural correlates of encoding in an incidental learning paradigm. Electroencephalogr Clin Neurophysiol, 1987, 67: 360-371

18 Sanquist T F, Rohrbaugh J W, Syndulko K, et al. Electrophysiological signs of levels of processing: perceptual analysis and recognition memory. Psychophysiology, 1980, 17: 568-576

19 Leynes P A, Marsh R L, Hicks J L, et al. Investigating the encoding and retrieval of intentions with event-related potentials. Conscious Cogn, 2003, 12: 1-30

20 Liu Y. Modern Chinese word frequency dictionary. Beijing: Yuhang Press, 1990

21 Guo C Y, Zhu Y, Ding J H, et al. An electrophysiological investigation of memory encoding, depth of processing, and word frequency in humans. Neurosci Lett, 2004, 356: 79-82

22 Otten L J, Sveen J, Quayle A H. Distinct patterns of neural activity during memory formation of nonwords versus words. J Cogn Neurosci, 2007, 19: 1776-1789

23 Guo C Y, Chen W J, Tian T, et al. Orientation to learning context modulates retrieval processing for unrecognized words. Chinese Sci Bull, 2010, 55: 2966-2973

24 Graf P, Uttl B. Prospective memory: a new focus for research. Conscious Cogn: An Int J, 2001, 10: 437-450

25 Maylor E A, Darby R J, Sala S D. Retrieval of performed versus to-be-performed tasks: a naturalistic study of the intention-superiority effect in normal aging and dementia. Appl Cogn Psychol, 2000, 14: 83-98

26 West R, Craik F I M. Age-related decline in prospective memory: The roles of cue accessibility and cue sensitivity. Psychol Aging, 1999, 14: 264-272

27 Taylor R S, Marsh R L, Hicks J L et al. The influence of par- tial-match cues on event-based prospective memory. Memory, 2004, 12: 203-213

28 Friedman D, Johnson R Jr. Event-related potential (ERP) studies of memory encoding and retrieval: A selective review. Micro Res Tech, 2000, 51: 6-28

29 Paller K A, Wagner A D. Observing the transformation of experience in to memory. Trends Cogn Sci, 2002, 6: 93-102

30 McDaniel M A, Robinson-Riegler B, Einstein G O. Prospective remembering: Perceptually driven or conceptual driven process? Mem Cogn, 1998, 26: 121-134

31 Okuda J, Fujii T, Yamadori A, et al. Participation of the prefrontal cortices in prospective memory: evidence from a PET study in humans. Neurosci Lett, 1998, 253: 127-130

32 Voss J L, Paller K A. Neural substrates of remembering-electroencephalographic studies. Learn Mem A Compr Ref, 2008, 3: 79-97

33 Guo C Y, Duan L, Li W, et al. Distinguishing source memory and item memory: Brain potentials at encoding and retrieval. Brain Res, 2006, 1118: 142-154

34 Fernández G, Weyerts H, Tendolkar I, et al. Event-related potentials of verbal encoding into episodic memory: Dissociation between the effects of subsequent memory performance and distinctiveness. Psychophysiology, 1998, 35: 709-720

35 Paller K A, McCarthy G, Wood C C. ERPs predictive of subsequent recall and recognition performance. Biol Psychol, 1988, 26: 269-276

36 Yovel G, Paller K A. The neural basis of the butcher-on-the-bus phenomenon: When a face seems familiar but is not remembered. Neuroimage, 2004, 21: 789-800

37 Liu R, Guo C Y, Jiang Y. An event-related potential study of working memory in children. Chinese Sci Bull, 2006, 51: 1467-1475

38 Meng Y F, Guo C Y. ERP dissociation and connection between implicit and explicit memory. Chinese Sci Bull, 2007, 52: 2945-2953

39 Nie A Q, Guo C Y, Shen M W. Material differences of auditory source retrieval: Evidence from event-related potential studies. Chinese Sci Bull, 2008, 53: 2801-2812

40 Liu Z M, Guo C Y. The interaction between working memory and selective attention. Chinese Sci Bull, 2007, 52: 1788-1795

Open Access This article is distributed under the terms of the Creative Commons Attribution License which permits any use, distribution, and reproduction in any medium, provided the original author(s) and source are credited. 